\title{
Universal Indestructibility is Consistent with Two Strongly Compact Cardinals
}

by

\author{
Arthur W. APTER \\ Presented by Czestaw BESSAGA
}

Summary. We show that universal indestructibility for both strong compactness and supercompactness is consistent with the existence of two strongly compact cardinals. This is in contrast to the fact that if $\kappa$ is supercompact and universal indestructibility for either strong compactness or supercompactness holds, then no cardinal $\lambda>\kappa$ is measurable.

In [2], the concepts of universal indestructibility for both strong compactness and supercompactness were introduced. Specifically, we say that universal indestructibility for supercompactness holds in a model $V$ for ZFC if every $V$-supercompact and partially supercompact (including measurable) cardinal $\delta$ has its degree of supercompactness fully Laver indestructible [7] under $\delta$-directed closed forcing. Analogously, universal indestructibility for strong compactness holds in a model $V$ for ZFC if every $V$-strongly compact and partially strongly compact (including measurable) cardinal $\delta$ has its degree of strong compactness fully indestructible under $\delta$-directed closed forcing. Readers are urged to consult [2] for further details.

One of the key results of [2] is Theorem 10, which states that if there are two supercompact cardinals, then universal indestructibility fails for partial supercompactness (and, as can be inferred from its proof, for partial strong compactness as well). In particular, if $\kappa$ is $\lambda^{+}$supercompact where $\lambda>\kappa$ is measurable, then universal indestructibility fails for both partial supercompactness and partial strong compactness. This is because under these

2000 Mathematics Subject Classification: 03E35, 03E55.

Key words and phrases: supercompact cardinal, strongly compact cardinal, indestructibility, universal indestructibility.

The author's research was partially supported by PSC-CUNY Grant 66489-00-35 and a CUNY Collaborative Incentive Grant. 
circumstances, it must be the case that there are unboundedly many in $\kappa$ cardinals $\delta<\kappa$ whose measurability can be destroyed by adding a Cohen subset of $\delta$. The proof, however, is heavily dependent on the fact that supercompactness embeddings are into highly closed inner models. Since strongly compact cardinals do not necessarily possess such embeddings, this leads to the following

QUESTION. Is universal indestructibility for either strong compactness or supercompactness consistent with the existence of more than one strongly compact cardinal?

The purpose of this note is to show that the answer to the above Question is yes. Specifically, we prove the following theorem.

Theorem 1. Suppose $V \vDash " Z F C+G C H+$ There is a high-jump cardinal". There is then a model of ZFC containing two strongly compact cardinals in which universal indestructibility for supercompactness holds. In addition, there is a model of ZFC containing two strongly compact cardinals in which universal indestructibility for strong compactness holds.

We note that the assumption of GCH is made for convenience and ease of presentation. At the end of this note, we will indicate how to prove Theorem 1 under arbitrary circumstances.

We recall from [2] that the cardinal $\kappa$ is a high-jump cardinal if there is an elementary embedding $j: V \rightarrow M$ having critical point $\kappa$ such that for some $\theta$ we have $M^{\theta} \subseteq M$ and $j(f)(\kappa)<\theta$ for every function $f: \kappa \rightarrow \kappa$. As Lemma 2 of [2] indicates, if $\kappa$ is almost huge, then $\kappa$ is the $\kappa$ th high-jump cardinal. Further, Lemma 3 of [2] tells us that if $\kappa$ is a high-jump cardinal, then $V_{\kappa} \vDash$ "ZFC + There is a proper class of supercompact cardinals". Thus, in terms of consistency strength, the property of being a high-jump cardinal lies strictly in between supercompactness and almost hugeness.

To prove Theorem 1, we combine the methods of [2] with the techniques of [1]. We begin, however, with the following lemma, which shows that the Lévy-Solovay results [8] of preservation of large cardinal properties under small forcing are true for high-jump cardinals.

Lemma 0.1. Suppose $V \vDash " Z F C+\kappa$ is a high-jump cardinal $+\mathbb{P}$ is a partial ordering such that $|\mathbb{P}|<\kappa$ ". Then $V^{\mathbb{P}} \vDash$ " $\kappa$ is a high-jump cardinal".

Proof. Let $j: V \rightarrow M$ and $\theta$ witness that in $V, \kappa$ is a high-jump cardinal. By standard arguments (see, e.g., the proof of the Main Theorem of [6]), since $|\mathbb{P}|<\kappa$, in $V^{\mathbb{P}}, j$ lifts to $j^{*}: V^{\mathbb{P}} \rightarrow M^{j(\mathbb{P})}$. Also, $M^{j(\mathbb{P})}$ remains $\theta$ closed with respect to $V^{\mathbb{P}}$. Thus, the proof of Lemma 0.1 will be complete once we have shown that $j^{*}$ and $\theta$ continue to witness that $\kappa$ is a high-jump cardinal in $V^{\mathbb{P}}$. 
To do this, let $p \in \mathbb{P}$ and $\dot{f}$ be such that $p \Vdash " \dot{f}: \kappa \rightarrow \kappa$ is a function". Define in $V$ a function $g: \kappa \rightarrow \kappa$ by $g(\alpha)=\sup (\{\beta<\kappa \mid$ For some $q$ extending $p, q \Vdash " \dot{f}(\alpha)=\beta "\})$. Since $|\mathbb{P}|<\kappa$ and $\kappa$ is regular, $g$ is well defined. It is then the case that $p \Vdash$ "For every $\alpha<\kappa, \dot{f}(\alpha) \leq g(\alpha)$ ", from which it can be immediately inferred that $M^{j(\mathbb{P})} \vDash " j^{*}(f)(\kappa) \leq j^{*}(g)(\kappa)=$ $j(g)(\kappa)<\theta$ ". This completes the proof of Lemma 0.1.

Given Lemma 0.1, it now becomes possible to prove Theorem 1. Suppose $V \vDash$ "ZFC $+\mathrm{GCH}+\kappa$ is a high-jump cardinal". By our earlier remarks, let $\lambda$ be the least cardinal such that $V_{\kappa} \vDash$ "ZFC $+\lambda$ is supercompact".

Working in $V_{\kappa}$, let $\mathbb{P}$ be the partial ordering of Theorem 1 of [1], defined with respect to $\lambda$. Since $V_{\kappa} \vDash \mathrm{GCH}$, the arguments of [1] show that $V_{\kappa}^{\mathbb{P}} \vDash$ "ZFC $+\lambda$ is both the least strongly compact and least measurable cardinal $+\lambda$ 's strong compactness is indestructible under $\lambda$-directed closed forcing". Since $\mathbb{P}$ may be defined so that $|\mathbb{P}|=\lambda<\kappa$, standard arguments show that GCH holds at $\lambda$ after forcing with $\mathbb{P}$. Further, by Lemma $0.1, V^{\mathbb{P}} \vDash$ " $\kappa$ is a high-jump cardinal".

Working now in $V^{\mathbb{P}}$, let $\mathbb{Q}$ be the partial ordering of either Theorem 5 or Theorem 6 of [2], with the first non-trivial stage of forcing taking place at or above the least $V^{\mathbb{P}}$-weakly compact cardinal $\sigma$ above $\lambda$. Let $\gamma<\kappa$ be the stage at which the construction of $\mathbb{Q}$ terminates, and let $\delta<\kappa$ be the least weakly compact cardinal above $\gamma$ in $V^{\mathbb{P} * \dot{\mathbb{Q}}}$. By the arguments of [2], $V_{\delta}^{\mathbb{P} * \dot{\mathbb{Q}}} \vDash$ "ZFC + Universal indestructibility for supercompactness holds for every measurable cardinal above $\lambda+\gamma$ is the least supercompact cardinal". Since the definition of $\mathbb{Q}$ from [2] ensures that $\mathbb{Q}$ is $\sigma$-directed closed in both $V^{\mathbb{P}}$ and $V_{\delta}^{\mathbb{P}}$, we may infer using $\lambda<\sigma<\gamma<\delta<\kappa$ that $V_{\delta}^{\mathbb{P} * \dot{\mathbb{Q}}} \vDash$ "GCH holds at $\lambda+\lambda$ is both the least strongly compact and least measurable cardinal $+\lambda$ 's strong compactness is indestructible under $\lambda$-directed closed forcing". However, since $V_{\delta}^{\mathbb{P} * \dot{\mathbb{Q}}} \vDash " 2^{\lambda}=\lambda^{+}+\lambda$ is the least measurable cardinal", we may immediately infer that $V_{\delta}^{\mathbb{P} * \dot{\mathbb{Q}}} \vDash " \lambda$ is not $2^{\lambda}=\lambda^{+}$ supercompact". Thus, since $\lambda$ is an indestructible strongly compact cardinal in $V_{\delta}^{\mathbb{P} * \dot{Q}}, V_{\delta}^{\mathbb{P} * \dot{\mathbb{Q}}} \vDash$ “' $\lambda$ 's degree of supercompactness (namely measurability) is indestructible". Hence, $V_{\delta}^{\mathbb{P} * \dot{\mathbb{Q}}}$ is a model of ZFC in which universal indestructibility for supercompactness holds and there are two strongly compact cardinals (namely $\lambda$ and $\gamma$ ). Finally, if we change the definition of $\mathbb{Q}$ to be the partial ordering of Theorem 7 of [2] but keep the meanings of $\lambda, \sigma, \gamma$, and $\delta$ as before, then by the arguments of $[2], V_{\delta}^{\mathbb{P} * \dot{\mathbb{Q}}} \vDash$ "ZFC + Universal indestructibility for strong compactness holds for every measurable cardinal above $\lambda+\gamma$ is the least strongly compact cardinal above $\lambda$ ". Since once again the definition of $\mathbb{Q}$ ensures that $\mathbb{Q}$ is $\sigma$-directed closed in both $V^{\mathbb{P}}$ and $V_{\delta}^{\mathbb{P}}$, 
$V_{\delta}^{\mathbb{P} * \dot{Q}} \vDash$ " $\lambda$ is an indestructible strongly compact cardinal". Consequently, $V_{\delta}^{\mathbb{P} * \dot{\mathbb{Q}}}$ is a model of ZFC in which universal indestructibility for strong compactness holds and there are two strongly compact cardinals (namely $\lambda$ and $\gamma$ ). This completes the proof of Theorem 1.

As was mentioned earlier, it is possible to prove Theorem 1 without the additional assumption of GCH. To see how this is done, note that the forcing $\mathbb{P}$ given above is a Gitik style iteration of Prikry-like forcings as described in [4] and [5]. Such iterations are possible regardless of any GCH assumptions in the ground model. We therefore must show that the arguments given in Theorem 1 of [1], which were presented using GCH, are possible when GCH does not necessarily hold.

That this can be accomplished is found by a close examination of the reasoning done in [1]. Theorem 1 of [1] is proven via Lemmas 1 and 2 of that paper, and the proof of Lemma 1 does not require GCH. As can be verified by examining its proof, any use of GCH in Lemma 2 of [1] may be replaced by choosing initially a large enough singular strong limit cardinal of sufficiently high cofinality satisfying $\mathrm{GCH}$, which is possible by Solovay's theorem of [9]. Thus, if we force with the partial ordering $\mathbb{P}$ of Theorem 1 over a model in which GCH is not necessarily true, we are still able to verify that the cardinal $\lambda$ of Theorem 1 is indestructibly strongly compact and the least measurable cardinal in $V_{\kappa}^{\mathbb{P}}$. If we then force $\mathrm{GCH}$ at $\lambda$ (if it does not already hold) by adding a Cohen subset of $\lambda^{+}, \lambda$ remains indestructibly strongly compact and the least measurable cardinal. The remainder of the proof of Theorem 1 then goes through as before.

Let us note that the partial ordering $\mathbb{P}$ for Theorem 1 does not force universal indestructibility for Ramseyness and weak compactness $\left({ }^{1}\right)$, as do the partial orderings of Theorems 6 and 7 of [2]. Thus, we can ask if universal indestructibility for Ramseyness and weak compactness, together with universal indestructibility for either supercompactness or strong compactness, is consistent with the existence of two or more strongly compact cardinals.

We remark that since the forcing of Theorem 1 of [1] adds bona fide Prikry sequences, by Theorem 11.1(1) of [3], this forcing adds non-reflecting stationary sets of ordinals of cofinality $\omega$. By Theorem 4.8 of [10] and the succeeding remarks, such a set of ordinals cannot exist above a strongly compact cardinal. Thus, the forcing $\mathbb{P}$ of Theorem 1 of this paper cannot be iterated in order to obtain a version of Theorem 1 in which there are more

$\left({ }^{1}\right)$ Universal indestructibility for Ramseyness holds in a model $V$ for ZFC if every $V$ Ramsey cardinal $\delta$ has its Ramseyness fully indestructible under $\delta$-directed closed forcing. Similarly, universal indestructibility for weak compactness holds in a model $V$ for ZFC if every $V$-weakly compact cardinal $\delta$ has its weak compactness fully indestructible under $\delta$-directed closed forcing. 
than two strongly compact cardinals. Since the methods of [2] by themselves do not allow for the construction of a model for universal indestructibility for either strong compactness or supercompactness containing more than one strongly compact cardinal, we conclude by asking if it is possible to have models for universal indestructibility for either strong compactness or supercompactness containing more than two strongly compact cardinals.

\section{References}

[1] A. Apter and M. Gitik, The least measurable can be strongly compact and indestructible, J. Symbolic Logic 63 (1998), 1404-1412.

[2] A. Apter and J. D. Hamkins, Universal indestructibility, Kobe J. Math. 16 (1999), 119-130.

[3] J. Cummings, M. Foreman and M. Magidor, Squares, scales, and stationary reflection, J. Math. Logic 1 (2001), 35-98.

[4] M. Gitik, Changing cofinalities and the nonstationary ideal, Israel J. Math. 56 (1986), 280-314.

[5] - On closed unbounded sets consisting of former regulars, J. Symbolic Logic 64 (1999), 1-12.

[6] J. D. Hamkins and W. H. Woodin, Small forcing creates neither strong nor Woodin cardinals, Proc. Amer. Math. Soc. 128 (2000), 3025-3029.

[7] R. Laver, Making the supercompactness of $\kappa$ indestructible under $\kappa$-directed closed forcing, Israel J. Math. 29 (1978), 385-388.

[8] A. Lévy and R. Solovay, Measurable cardinals and the continuum hypothesis, ibid. 5 (1967), 234-248.

[9] R. Solovay, Strongly compact cardinals and the GCH, in: Proceedings of the Tarski Symposium, Proc. Sympos. Pure Math. 25, Amer. Math. Soc., Providence, 1974, 365-372.

[10] R. Solovay, W. Reinhardt and A. Kanamori, Strong axioms of infinity and elementary embeddings, Ann. Math. Logic 13 (1978), 73-116.

Arthur W. Apter

Department of Mathematics

Baruch College of CUNY

New York, NY 10010, U.S.A. E-mail: awabb@cunyvm.cuny.edu

Web: http://faculty.baruch.cuny.edu/apter 